\title{
Central Hepatectomy Using the Hilar Approach for Removal of Tumors in the Paracaval Portion of the Caudate Lobe
}

\author{
Harufumi Maki ${ }^{1}$, Yoshihiro Sakamoto ${ }^{2}$, Hiroji Shinkawa ${ }^{3}$, Yusuke Kazami ${ }^{1}$, Junichi Arita ${ }^{1}$, \\ Nobuhisa Akamatsu ${ }^{1}$, Junichi Kaneko ${ }^{1}$, Norihiro Kokudo ${ }^{4}$ and Kiyoshi Hasegawa ${ }^{1}$
}

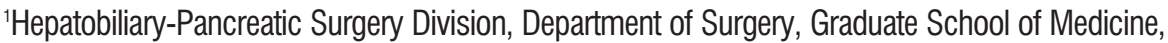
University of Tokyo, Tokyo, Japan

2Department of Surgery, Kyorin University, School of Medicine

${ }^{3}$ Department of Hepato-Biliary-Pancreatic Surgery, Osaka City University,

Graduate School of Medicine

${ }^{4}$ Department of Surgery, National Center for Global Health and Medicine

\section{ABSTRACT}

Safe hepatectomy for the paracaval portion of the caudate lobe is technically demanding. We propose central hepatectomy using the hilar approach for resection of the paracaval portion. The right liver with the caudate lobe was totally mobilized from the inferior vena cava. Central hepatectomy with or without middle hepatic vein (MHV) resection was performed by dividing the right anterior and left medial sections vertically toward the inferior vena cava. The bilateral Glissonian pedicles were widely exposed (hilar approach), and the liver parenchyma including the paracaval portion of the liver was removed with a good surgical view.This procedure was performed in four patients with hepatocellular carcinoma, one patient with intrahepatic cholangiocarcinoma, and one patient with colorectal liver metastasis. Combined resection of the MHV was performed in four patients, and removal of the tumor thrombus in the bile duct was performed in one patient. The median operation time was 439 minutes, and the median blood loss was $800 \mathrm{ml}$. The resected liver parenchyma was $12.0 \%$ (range, $4.7 \%-39.5 \%$ ) of the total liver volume. No patients developed postoperative liver failure. Central hepatectomy using the hilar approach is a safe, parenchyma-preserving surgical procedure for removal of tumors located in the paracaval portion.

Key words: central hepatectomy, hilar approach, caudate lobe, paracaval tumor, parenchymapreserving hepatectomy

\section{INTRODUCTION}

The caudate lobe is located deep within the liver and surrounded by the three major hepatic veins, the inferior vena cava (IVC), and the hepatic hilum $(1,2)$. The caudate lobe of the liver consists of three portions: the Spiegel lobe, the caudate process, and the paracaval portion $(3,4)$. Among the three portions of the caudate lobe, resection of tumors in the paracaval portion is most challenging. Resection of hepatocellular carcinoma (HCC) in the paracaval portion is reportedly associated with a longer operation time, larger blood loss
Corresponding author: Kiyoshi Hasegawa, MD 7-3-1 Hongo, Bunkyo-ku, Tokyo Japan 113-8655

Tel: +81-3-3815-5411

Fax: +81-3-5684-3989

E-mail: kihase-tky@umin.ac.jp

\author{
Abbreviations: \\ MHV - middle hepatic vein \\ IVC - inferior vena cava \\ HCC - hepatocellular carcinoma \\ BDTT - bile duct tumor thrombus \\ $\mathrm{RHV}$ - right hepatic vein
}

Received: 30.06 .2019

Accepted: 28.07.2019 
volume, and smaller surgical margins than resection in other sites within the caudate lobe (5). Resection of the paracaval portion can be performed in combination with other resection techniques such as extended hemihepatectomy (6) or posterior sectionectomy in patients with enough functional reserve (7). However, isolated caudate lobectomy using a high dorsal approach (8) or anterior transhepatic approach (9) is required in patients with impaired liver function reserve, and the liver parenchyma should be preserved as much as possible to prevent postoperative liver failure. Although the high dorsal approach is ultimately an isolated caudate lobectomy procedure, it is technically demanding because of the strictly limited surgical field. The transhepatic approach is also a parenchyma-preserving alternative, but removal of tumors invading the middle hepatic vein (MHV) is difficult, and some ischemic or congested areas remain.

Therefore, we propose central hepatectomy using the hilar approach as a useful and safe alternative for removal of tumors located in the paracaval portion of the liver with a good surgical field.

\section{CASE REPORTS}

Central hepatectomy using the hilar approach was performed in six patients: four with HCCs in segment 1 [one of these patients had HCC with a bile duct tumor thrombus (BDTT) via the anterior bile duct (Patient \#2)], one patient with intrahepatic cholangiocarcinoma, and one patient with multiple colorectal liver metastases in segments 4,5 , and 8 and the paracaval portion. The patients' characteristics are shown on table 1 . We calculated the future liver remnant from the preoperative computed tomography images using threedimensional simulation software (Synapse Vincent; Fujifilm, Tokyo, Japan) (10). The surgical indication was determined based on our resection criteria as represented by the indocyanine green retention rate at $15 \min (11)$.

\section{SURGICAL PROCEDURE}

An inverted L-shaped skin incision was used to perform the laparotomy. In Patient \#3, a thoracotomy was added at the ninth intercostal space because a sufficient surgical field was not obtained. The location of the tumor was confirmed by intraoperative ultrasonography. First, the right liver with the caudate lobe was totally mobilized from the IVC by ligating and dividing the short hepatic veins. The right hepatic vein (RHV) was taped as a landmark during liver transection. The IVC was also taped to control bleeding during resection of the liver parenchyma, if necessary. Second, liver transection lines were designed to remove the central part of the liver, including the paracaval portion. The left-side line was designed along the falciform ligament in case of combined resection of the MHV or Spiegel lobe. In contrast, the left-side line might course along the MHV if required according to the tumor location (fig. $1 a-d)$. To determine the right-side line, indigocarmine solution with indocyanine green was injected into the portal branch of the right anterior section or the ventral branch of segment 8 , and the stained area was marked with electrocautery $(12,13)$ (fig. $2 a, b)$. To determine the right-sided border of the

Table 1 - Characteristics and surgical outcomes of central hepatectomy using the hilar approach

\begin{tabular}{|c|c|c|c|c|c|c|c|c|c|c|c|c|}
\hline No. & $\begin{array}{l}\text { Age } \\
\text { Sex }\end{array}$ & Diagnosis & $\begin{array}{l}\text { Tumor } \\
\text { size } \\
\text { (cm) }\end{array}$ & $\begin{array}{c}\text { ICG-R15 } \\
(\%)\end{array}$ & $\begin{array}{l}\text { Procedure of } \\
\text { central } \\
\text { hepatectomy }\end{array}$ & $\begin{array}{c}\text { Op time } \\
\text { (min) }\end{array}$ & $\begin{array}{l}\text { Blood } \\
\text { loss } \\
\text { (mL) }\end{array}$ & Transfusion & $\begin{array}{l}{ }^{*} \text { Resected } \\
\text { liver volume } \\
\text { (mL) }(/ \mathrm{TLV})\end{array}$ & Morbidity & $\begin{array}{c}\text { Hospital } \\
\text { stay } \\
\text { (days) }\end{array}$ & Outcome \\
\hline 1 & $\begin{array}{c}80 \text { 's } \\
F\end{array}$ & $\mathrm{HCC}$ & 2.5 & 9.8 & S4+S8vent+PC+SP with MHV resection & 442 & 110 & no & $220(25.7 \%)$ & None & 14 & $\begin{array}{l}\text { No recurrence, } \\
\text { alive }(4 \mathrm{y} 4 \mathrm{~m})\end{array}$ \\
\hline 2 & $\begin{array}{c}\text { 60's } \\
M\end{array}$ & $\begin{array}{l}\text { HBV, HCC } \\
\text { with BDTT }\end{array}$ & 3 & 22.8 & $\begin{array}{l}\text { Partial S5+S8vent+PC+CP } \\
\text { with T-tube drainage }\end{array}$ & 420 & 1100 & no & $63(6.8 \%)$ & $\begin{array}{l}\text { Bile } \\
\text { leakage }\end{array}$ & 33 & $\begin{array}{l}\text { No recurrence, } \\
\text { alive }(3 y 1 \mathrm{~m})\end{array}$ \\
\hline 3 & $\begin{array}{l}70 \text { 's } \\
M\end{array}$ & $\mathrm{HCC}$ & 13 & 17 & S4+S5+S8vent+PC with MHV resection & 715 & 3380 & no & $87(4.7 \%)$ & Ascites & 19 & $\begin{array}{l}\text { Liver recurrence, } \\
\text { alive }(1 \mathrm{y} 1 \mathrm{~m})\end{array}$ \\
\hline 4 & $\begin{array}{l}80 \text { 's } \\
\mathrm{F}\end{array}$ & $\mathrm{HCV}, \mathrm{HCC}$ & 1.7 & 15.8 & S8vent+PC & 433 & 1115 & yes & $39(5.2 \%)$ & $\begin{array}{l}\text { Pulmonary } \\
\text { effusion }\end{array}$ & 13 & $\begin{array}{l}\text { No recurrence, } \\
\text { alive (1y11m) }\end{array}$ \\
\hline 5 & 60's & ICC & 9.5 & 7.0 & S4/5/8+PC with MHV resection & 435 & 500 & no & $359(39.5 \%)$ & $\begin{array}{l}\text { Abdominal } \\
\text { abscess }\end{array}$ & 25 & $\begin{array}{l}\text { Liver recurrence, } \\
\text { alive }(1 \mathrm{y} 5 \mathrm{~m})\end{array}$ \\
\hline 6 & $\begin{array}{c}30 \text { 's } \\
M\end{array}$ & CRLM & 2.5 & 9.5 & Partial S4/5/8 +PC with MHV resection & 477 & 450 & no & $143(17.2 \%)$ & None & 14 & $\begin{array}{l}\text { Pelvis, liver } \\
\text { recurrence, alive } \\
(3 \mathrm{y} 0 \mathrm{~m})\end{array}$ \\
\hline
\end{tabular}

F, female; M, male; HCC, hepatocellular carcinoma; BDTT, bile duct tumor thrombus; ICC, intrahepatic cholangiocarcinoma; CRLM, colorectal liver metastasis; ICG-R15, indocyanine green retention rate at 15 min; vent, ventral area; PC, paracaval portion; SP, Spiegel lobe; MHV, middle hepatic vein; CP, caudate processs; TLV, total non-cancerous liver volume; LN, lymph node

*'Resected liver volume'= 'Resected volume of the specimen' - 'Tumor volume calculated by CT volumetry' 


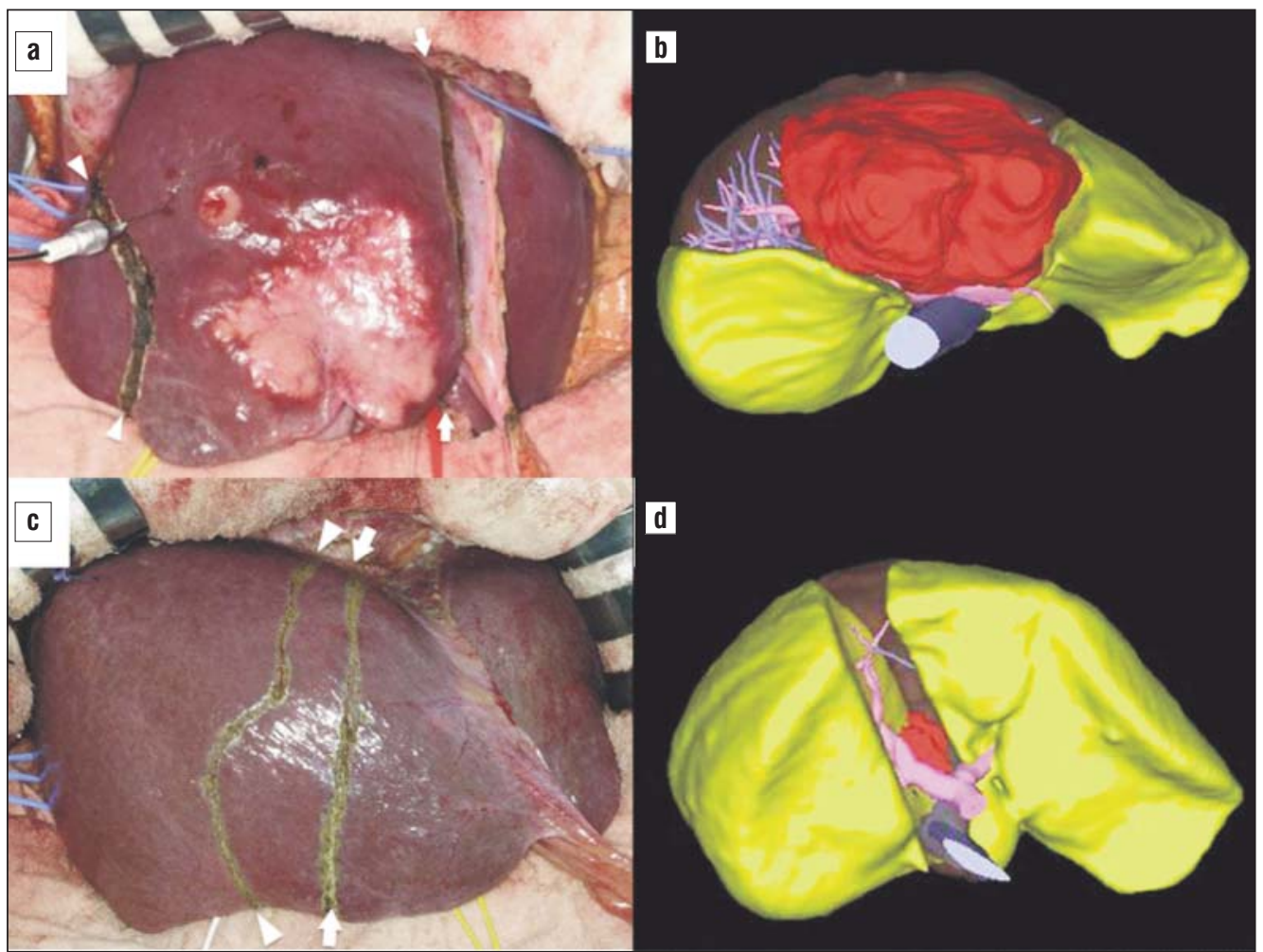

Figure 1 - Liver resection lines in central hepatectomy

a. The tumor of Patient \#5 was large, requiring combined resection of the MHV. The left-side line was along the falciform ligament (arrows). The right-side line was marked between the right anterior section and the right posterior section (arrowheads). b. The caudal view of a three-dimensional simulation image of Patient \#5 is shown. The tumor and the future liver remnant are colored red and yellow, respectively. c. The liver function of Patient \#2 was impaired, requiring preservation of a large future liver remnant. The left-side line was marked along the MHV (arrows). The right-side line was determined by the location of the anterior Glissonian pedicle and the root of the RHV (arrowheads). d. The caudal view of a three-dimensional simulation image of Patient \#2 is shown. The tumor and the future liver remnant are colored red and yellow, respectively. MVH, middle hepatic vein; RHV, right hepatic vein

paracaval portion, the paracaval branch was punctured when possible (fig. $2 c, d)$. Third, liver transection was started on the right side, dividing the portal branches associated with right anterior section or dividing the right anterior fissure, exposing the wall of the RHV. Liver transection was performed using the clamp-crush technique under the Pringle maneuver. The left hand of the operator was placed behind the right liver and in front of the IVC, pushing up and controlling the venous backflow from the RHV. The parenchyma belonging to the paracaval portion was easily divided with guidance provided by the left hand between the right liver and IVC or using the landmark of the taped RHV (fig. $3 a, b$ ). The caudate branches originating from the hilar plate were meticulously ligated and divided, allowing dissection of the caudate lobe off the hilar plate (fig. $4 a, b)$. During these maneuvers, the bilateral Glissonian pedicles were taped and the hilar plate was widely exposed (hilar approach). Next, the left side of the liver was transected by dividing the portal pedicles supplying the left medial section (segment 4) toward the root of the MHV. Arantius' ligament was divided, but removal of the Spiegel lobe was required in selected cases. Finally, the MHV was divided and the central liver with the caudate lobe was extracted. The stump of the MHV was sutured continuously. The cut surfaces after central hepatectomy and the resected specimen are shown in fig. 5 .

\section{RESULTS}

The surgical outcomes of all six patients (three men, three women) are summarized in table 1 . The patients' median age was 71 years (range, 34-83 years). The median tumor size was $2.8 \mathrm{~cm}$. The median indocyanine green retention rate at 15 min was $12.8 \%$. Combined resection with the MHV was performed in four patients, and the MHV was preserved in two patients. The median operation time was $439 \mathrm{~min}$, and the median blood loss volume was $800 \mathrm{~mL}$. The median occlusion time during the Pringle maneuver was 118 min (range, 60-143 $\mathrm{min}$ ). The median resected volume 


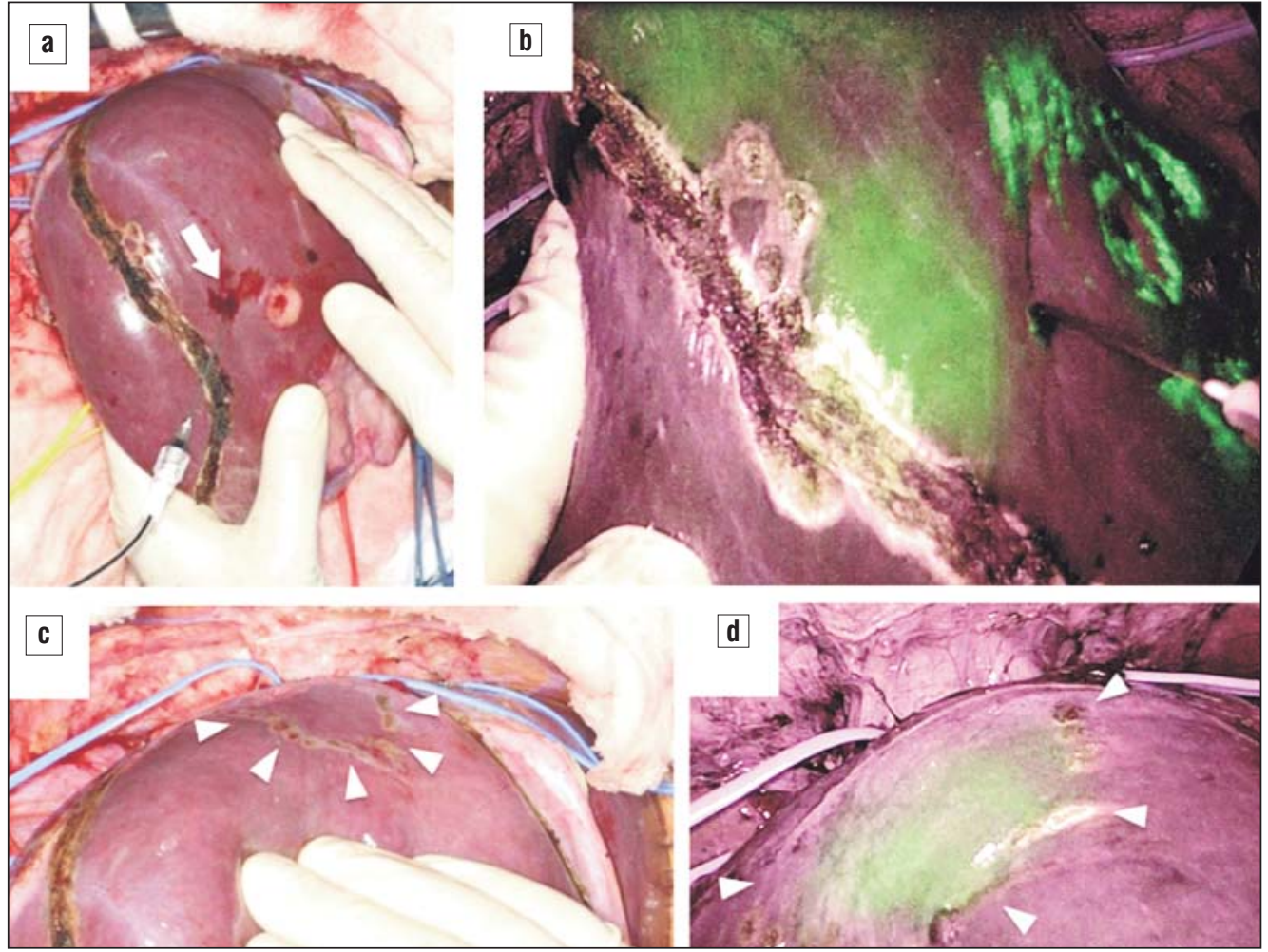

Figure 2 - An example of identification of the anatomical border (Patient \#5)

a. The portal branch of the right anterior section was punctured, and indigocarmine solution with indocyanine green was injected (arrow). b. The area stained by indocyanine green appeared green by the fluorescence imaging system (PINPOINT; Novadaq, Mississauga, Ontario, Canada). c. Indigocarmine solution with indocyanine green was also injected into the portal branch of the paracaval portion. However, the border of the paracaval portion was obscure (arrowheads). d. The border of the paracaval portion was clearly visualized by the PINPOINT system (arrow heads).

was $12.0 \%$ (range, $4.7 \%-39.5 \%$ ) of the total liver. No patients developed postoperative liver failure.

\section{DISCUSSIONS}

We modified the isolated caudate lobectomy procedure using an anterior approach and described central hepatectomy using the hilar approach,

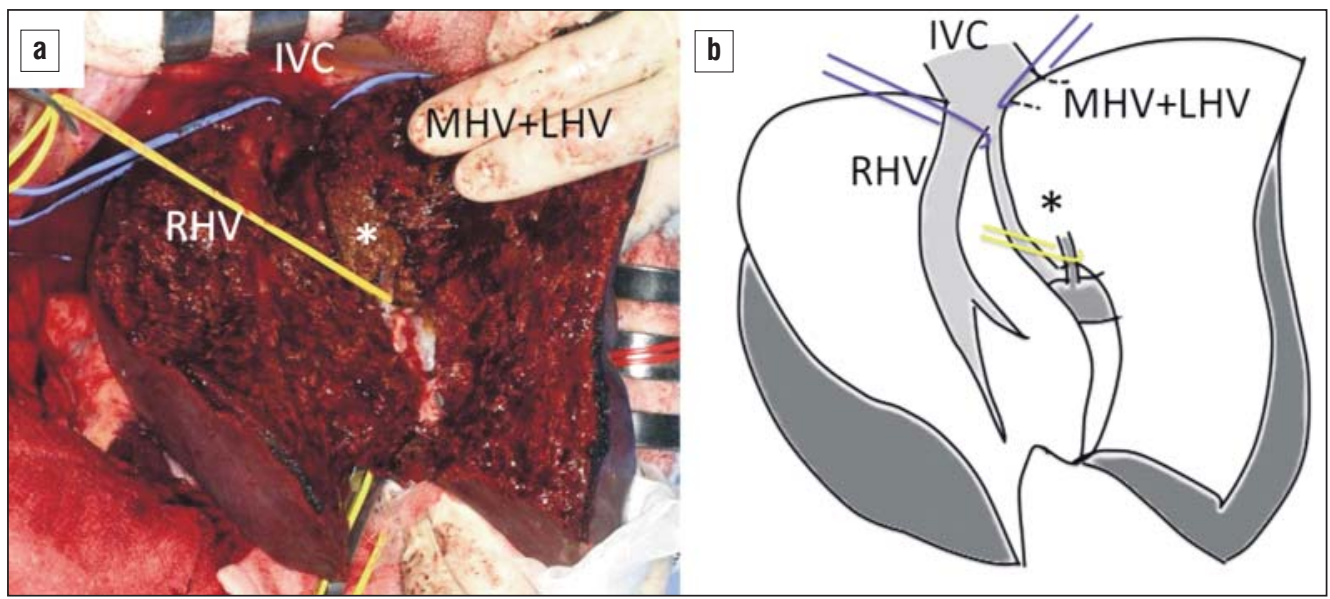

Figure 3 - An example of the cut surface of the right side of central hepatectomy (Patient \#5)

a. The root of the RHV and the common root of the MHV and LHV were taped by blue vessel loops. The right anterior section was divided vertically until the wall of the IVC was exposed. The portal branch of the paracaval portion was taped by a yellow vessel loop. The paracaval portion (asterisk) was included in the excision side. $b$. A schema of figure $3 a$ is shown. IVC, inferior vena cava; RHV, right hepatic vein; MHV, middle hepatic vein; LHV; left hepatic vein 


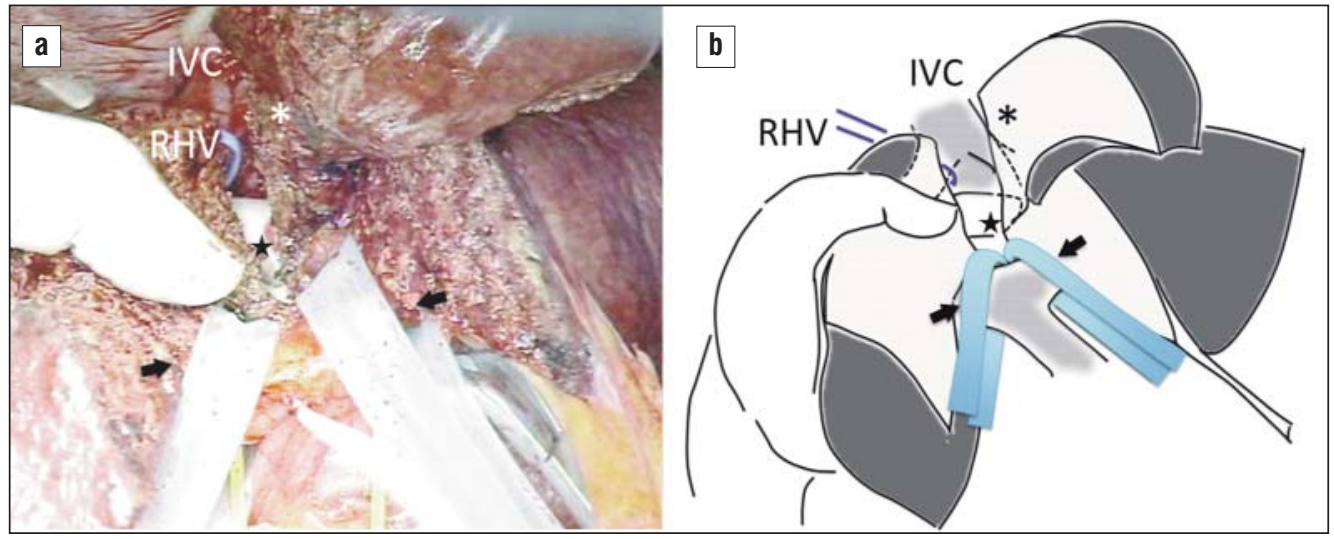

Figure 4 - Tips for central hepatectomy using the hilar approach (Patient \#1)

a. The bilateral Glissonian pedicles were taped (arrows) and the hilar plate was pulled caudally for a good surgical view. The surgeon's left index finger (star) was placed on the ventral side of the IVC, the dorsal side of the paracaval portion (asterisk), the caudal side of the root of the RHV, and the cranial side of the hilar plate. This procedure facilitated bleeding control. b. A schema of figure 4 a is shown. IVC, inferior vena cava; RHV, right hepatic vein

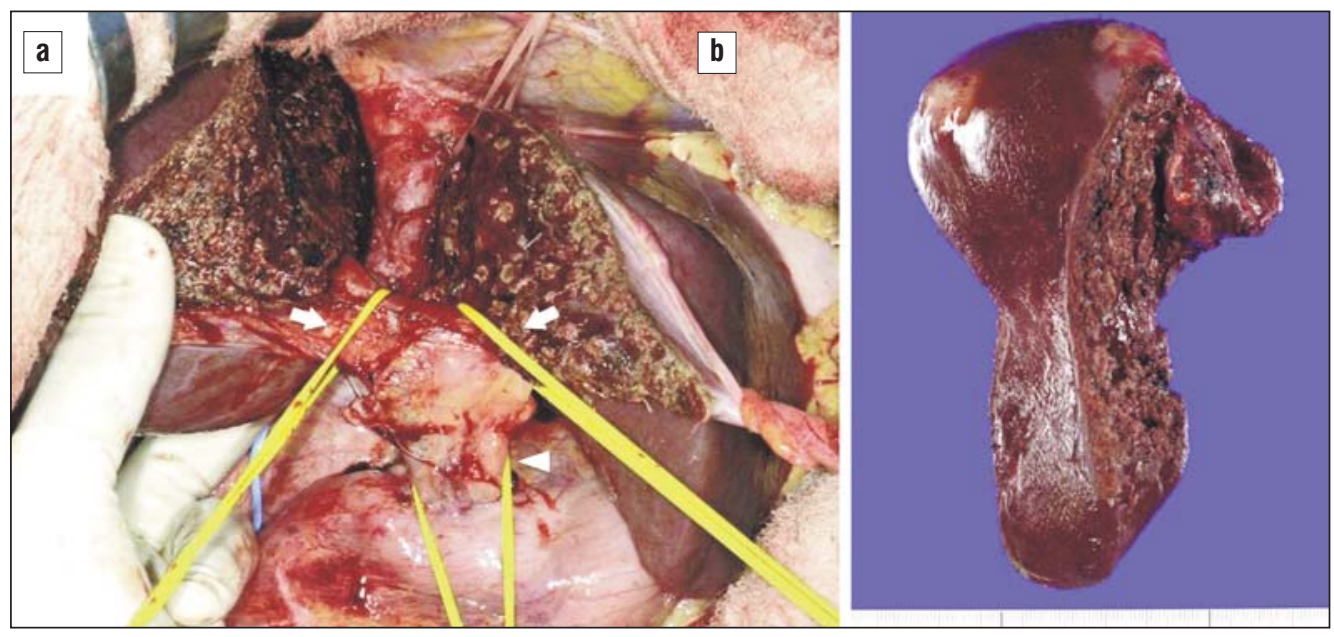

Figure 5.

a. The cut surfaces of central hepatectomy using the hilar approach are shown (Patient \#1). The bilateral Glissonian pedicles (arrows) and the hepatoduodenal ligament (arrowhead)were taped. b. An example of the resected specimen is shown (Patient \#6).

especially for removal of tumors located in the paracaval portion of the liver with preservation of the greatest amount of liver parenchyma possible. This technique has two main differences from the anterior approach: all or part of the anterior section and left medial section are sacrificed in a square shape, and the MHV is divided if necessary to obtain a good surgical field, especially behind the MHV.

Central hepatectomy using the hilar approach can maintain a good balance between establishment of a favorable surgical field and preservation of the remnant liver function. Longitudinal and straight transection lines can ensure technical ease and a good surgical field. Additionally, our case series included one patient (Patient \#3) whose tumor was $>10 \mathrm{~cm}$ in size and another patient (Patient \#2) who underwent bile ductpreserving surgery for HCC with BDTT (14). By taping the bilateral Glissonian pedicles, the tumor was safely detached from the right Glissonian pedicle and the BDTT was removed from the right hepatic duct. Shindoh et al. (15) reported a similar technique for central hepatectomy. They resected the central segments systematically ( $88+$ S5 ventral area and S4), which required removal of $30 \%$ of the liver parenchyma. In contrast, the resected liver volume in our study was only $12 \%$ of the total liver volume, which could be indicated in patients with worse functional liver reserve. The transection line to the hepatic hilum can thus be chosen according to the patient's liver function (fig. 1, table 1). 
The first key to this approach is complete mobilization of the caudate lobe from the IVC and insertion of the left hand behind the right liver during transection of the right anterior section. This provides the surgeon with a good surgical view and makes it easy to control the venous bleeding from the hepatic veins by lifting the left hand. If the adhesions are too severe to mobilize the caudate lobe because of repeated liver resections, transhepatic enucleation may be a feasible option (16). The second key is to approach the hilar plate. That is, the bilateral Glissonian pedicles are taped and the hilar plate is widely exposed. The portal pedicles branching from the hilar plate can then be confidently ligated and divided. The perihilar Glissonian approach, also called "the taping game," is another effective method to achieve taping of the hilar plate (17). This approach was originally proposed by Takasaki et al. (18) and can enable taping of the hilar plate before liver transection (19). However, this Glissonian approach is not recommended when the paracaval tumor is suspected to invade the hepatic hilum or be accompanied by a tumor thrombus, as in Patient \#2 of the present series.

The paracaval portion reaches the diaphragmatic surface in almost half of patients based on anatomical studies using casts $(1,2,20)$ and three-dimensional computed tomography scans (21). Therefore, it would be reasonable to open the diaphragmatic surface of the right liver to completely remove the paracaval portion. Higaki et al. (7) reported the feasibility of combined resection of the paracaval portion together with segment 8 , especially when the tumor is located beyond the caval portion. In the present study, the dorsal part of the segment 8 was preserved in 5 of 6 patients (83\%). Surgeons can approach the paracaval portion even if they do not completely remove segment 8. Mesohepatectomy is another option for removal of tumors located at both the central bisections (22-24). However, these previous mesohepatectomy procedures were rarely intended to remove tumors in the paracaval portion (25). Another possible procedure for paracaval tumors is the "liver tunnel" method $(26,27)$, by which the tumor is hollowed through the liver parenchyma overlying the paracaval portion. However, the surgical field becomes deep and restricted by the surrounding liver parenchyma overlying the hepatic hilum. Tani et al. (28) reported resections of deeply located tumors in combination with adjacent segments, such as segment 6,7 , or 8 , to preserve the liver parenchyma. This approach is very effective when the tumor does not invade the major hepatic veins or hepatic hilum. Our central hepatectomy technique using the hilar approach is widely applicable among patients with centrally located tumors, even those that invade the MHV or hepatic hilum, and even in patients with poor functional reserve when a good surgical field is established by opening the central area of the liver using the pivot of the hepatic hilum.

Another issue regarding this procedure is the rightsided transection line of the paracaval portion. The right-sided boundary of the paracaval portion is not easy to confirm (29). However, three-dimensional imaging software recently revealed that a small branch from the RHV can be a landmark for the right-sided transection line in about half of patients (21).

\section{CONCLUSIONS}

Central hepatectomy using the hilar approach is a safe surgical procedure for paracaval tumors. Even for tumorsthat require MHV resection or tumor thrombectomy, our procedure can provide an adequately wide surgical view.

\section{Conflict of Interest}

The authors declare no conflict of interest.

\section{Sources of Financial Support}

There is no financial support on this study.

\section{Informed Consent}

Informed consent was obtained from all participants to be included in the study.

\section{REFERENCES}

1. Healey JE Jr, Schroy PC. Anatomy of the biliary ducts within the human liver; analysis of the prevailing pattern of branchings and the major variations of the biliary ducts. AMA Arch Surg 1953; 66(5):599-616.

2. Couinaud C, Delmas A, Patel J. Le Foie: Etudes Anatomiqueset Chirurgicales. Paris: Masson \&Cie; 1957.

3. Kumon M. Anatomy of the caudate lobe with special reference to portal vein and bile duct. (in Japanese with English abstract). Acta Hepatol Jpn 1985;26:1193-9.

4. Kumon M. Anatomical study of the caudate lobe with special reference to portal venous and biliary branches using corrosion liver casts and clinical application. Liver Cancer 2017;6(2):161-70.

5. Sakamoto Y, Nara S, Hata S, Yamamoto Y, Esaki M, Shimada K, et al. Prognosis of patients undergoing hepatectomy for solitary hepatocellular carcinoma originating in the caudate lobe. Surgery 2011;150(5):959-67.

6. Hawkins WG, DeMatteo RP, Cohen MS, Jarnagin WR, Fong Y, D'Angelica M, et al. Caudate hepatectomy for cancer: a single institution experience with 150 patients. J Am Coll Surg 2005; 200(3):345-52. 
7. Higaki T, Takayama T, Midorikawa Y. Ventral approach for resecting hepatocellular carcinoma in the caval portion of the caudate lobe. Surgery 2018;163(6):1245-9.

8. Takayama T, Tanaka T, Higaki T,Katou K, Teshima Y, Makuuchi M. High dorsal resection of the liver. J Am CollSurg 1994;179(1):72-5.

9. Yamamoto J, Kosuge T, Shimada K, Yamasaki S, Takayama T, Makuuchi M. Anterior transhepatic approach for isolated resection of the caudate lobe of the liver. World J Surg 1999;23(1):97-101.

10. Mise Y, Hasegawa K, Satou S, Shindoh J, Miki K, Akamatsu N, et al. How has virtual hepatectomy changed the practice of liver surgery? Experience of 1194 virtual hepatectomy before liver resection and living donor liver transplantation. Ann Surg 2017;268(1):127-33.

11. Imamura H, Seyama Y, Kokudo N,Maema A, Sugawara Y, Sano K, et al. One thousand fifty-six hepatectomies without mortality in 8 years. Arch Surg 2003;138(11):1198-206; discussion 1206.

12. Makuuchi M, Hasegawa H, Yamazaki S. Ultrasonically guided subsegmentectomy. SurgGynecolObstet 1985;161(4):346-50.

13. Inoue Y, Arita J, Sakamoto T, Ono Y, Takahashi M, Takahashi Y, et al. Anatomical liver resections guided by 3-dimensional parenchymal staining using fusion indocyanine green fluorescence imaging. Ann Surg 2015;262(1):105-11.

14. Yamamoto S, Hasegawa K, Inoue Y, Shindoh J, Aoki T, Sakamoto Y, et al. Bile duct preserving surgery for hepatocellular carcinoma with bile duct tumor thrombus. Ann Surg 2015;261(5):e123-5.

15. Shindoh J, Nishioka Y, Hashimoto M. Bilateral anatomic resection of the ventral parts of the paramedian sectors of the liver with total caudate lobectomy for deeply/centrally located liver tumors: a new technique maximizing both oncological and surgical safety. J HepatobiliaryPancreatSci 2017;24(12):E10-6.

16. Ishizawa T,Hasegawa K,lkeda M, Aoki T, Sano K, Imamura H, et al.Transhepatic approach for a small paracaval tumor in repeat resection.Dig Surg2007;24(6):409-12.

17. Figueroa $R$, Laurenzi A, Laurent A, Cherqui D. PerihilarGlissonian approach for anatomical parenchymal sparing liver resections: technical aspects: the taping game. Ann Surg 2018;267(3):53743.

18. Takasaki K, Kobayashi S, Tanaka S, Saito A, Yamamoto M, Hanyu F. Highly anatomically systematized hepatic resection with Glissonean sheath code transection at the hepatic hilus. Int Surg 1990;75(2):73-7.
19. Yamamoto M, Takasaki K, Ohtsubo T, Katsuragawa H, Fukuda C, Katagiri S. Effectiveness of systematized hepatectomy with Glisson's pedicle transection at the hepatic hilus for small nodular hepatocellular carcinoma: retrospective analysis. Surgery 2001;130(3):443-8.

20. Kwon D, Murakami G, Hata F, Wang HJ, Chung MS, Hirata K. Location of the ventral margin of the paracaval portion of the caudate lobe of the human liver with special reference to the configuration of hepatic portal vein branches. ClinAnat 2002; 15(6):387-401.

21. Maki H, Sakamoto Y, Kawaguchi Y, Akamatsu N, Kaneko J, Arita J, et al. Anatomical boundary between the caudate lobe of the liver and adjacent segments based on three-dimensional analysis for precise resections. J Gastrointest Surg 2018;22(10):1709-14.

22. McBride CM, Wallace S. Cancer of the right lobe of the liver: a variety of operative procedures. Arch Surg 1972;105(2):289-96.

23. Hasegawa H, Makuuchi M, Yamazaki S, Gunvén P. Central bisegmentectomy of the liver: experience in 16 patients. World J Surg 1989;13(6):786-90.

24. Mehrabi A, Mood ZA, Roshanaei N, Fonouni H,Müller SA, Schmied $\mathrm{BM}$, et al. Mesohepatectomy as an option for the treatment of central liver tumors. J Am CollSurg 2008;207(4):499-509.

25. Ishii $\mathrm{H}$, Ogino $\mathrm{S}$, Ikemoto $\mathrm{K}$, Toma $\mathrm{A}$, Nakamura $\mathrm{K}$, Itoh $\mathrm{T}$, et al. Mesohepatectomy with total caudate lobectomy of the liver for hepatocellular carcinoma. World J SurgOncol 2013;11:82.

26. Torzilli G, Palmisano A, Procopio F, Cimino M, Botea F, Donadon M, et al. A new systematic small for size resection for liver tumors invading the middle hepatic vein at its caval confluence: mini-mesohepatectomy. Ann Surg 2010;251(1):33-9.

27. Torzilli G, Cimino M, Procopio F, Costa G, Donadon M, Del Fabbro D, et al. Conservative hepatectomy for tumors involving the middle hepatic vein and segment 1: the liver tunnel. Ann Surg Oncol 2014; 21(8):2699.

28. Tani K, Ishizawa T, Sakamoto Y, Hasegawa K, Kokudo N. Surgical approach to "right hepatic core": deepest region surrounded by major portal pedicles and right hepatic vein. Dig Surg 2018; 35(4):350-8.

29. Kitagawa S, Murakami G, Hata F, Hirata K. Configuration of the right portion of the caudate lobe with special reference to identification of its right margin. Clin Anat 2000;13(5):321-40. 